\title{
THE GHANAIAN WOMAN'S EXPERIENCE AND PERCEPTION OF ULTRASOUND USE IN ANTENATAL CARE
}

\author{
Y. B. MENSAH ${ }^{1}$, K. NKYEKYER ${ }^{2}$ and K. MENSAH ${ }^{3}$ \\ ${ }^{1}$ Departtment of Radiology, Korle Bu Teaching Hospital, P. O. Box KB 77, Korle Bu Accra, Ghana \\ ${ }^{2}$ Department of Obstetrics and Gynaecology University of Ghana Medical School, Korle Bu, Accra, Ghana \\ ${ }^{3}$ P. O Box GP 15533 Kumasi
}

DOI: http://dx.doi.org/10.4314/gmj.v48i1.5

Corresponding Author: Dr. Y.B. Mensah

Email: ybmensah@yahoo.com

Conflict of Interest: None declared

\section{SUMMARY}

Objective: To evaluate how Ghanaian women perceive the use, and their assessment of the experience, of antenatal ultrasound scanning.

Design: Cross-sectional study, using intervieweradministered questionnaire, from 25th February to 16th April, 2011

Setting: Obstetrics Units of Korle Bu Teaching Hospital, Accra, University of Ghana Hospital, Legon and Ga South District Hospital, Weija.

Methods: A total of 337 clients were randomly selected after delivery and interviewed. Issues addressed included why women went for antenatal ultrasound, their knowledge of the uses of ultrasound in pregnancy, information provided by health care providers, clients' eagerness to know the sex of their fetuses, and their overall assessment of the ultrasound scanning experience.

Results: The mean number of scans was 2.2(1.1). Most were performed on the request of a doctor or midwife; $154(45.7 \%)$ were not told the reasons for the request and $185(54.9 \%)$ did not have the results explained to them. For $239(70.9 \%)$ women the sonographer did not explain the procedure before the examination; $89(26.4 \%)$ were allowed to ask questions and $61(18.1 \%)$ were allowed to see their fetuses on the monitor. One hundred and sixty respondents (47.5\%) asked for and were told the sex of their fetuses, with accuracy at delivery of $86.5 \%$.

Conclusion: Most respondents perceived antenatal ultrasound as a useful tool. There is lack of information flow from health care providers to clients concerning the indications for the ultrasound, the processes involved and the results of the procedure. Improvements in these areas are needed to enhance the experience of antenatal ultrasound among Ghanaian women.

Keywords: ultrasound examination, pregnancy, antenatal, Ghana

\section{INTRODUCTION}

Ultrasound imaging now forms an essential part of antenatal care around the world. The facility is now available in most government and private health facilities in developing countries. ${ }^{1}$ Antenatal ultrasound is a safe technology that meets the WHO guidelines for the use of technology (1994) because it is scientifically sound, accessible, affordable and acceptable. ${ }^{2,3}$ The use of ultrasound in pregnancy has changed from just listening to fetal heart sounds (sonicaid) to detecting very small fetal morphological defects. Presently, increased sophistication in the ultrasound technology allows mothers to see their fetuses in 3D or 4D reconstruction on monitors and know their sex long before the child is born. $^{4}$

There is some concern, however, that there may be overuse of the technology; in a study of the utilization of sonography in a periurban health centre in Uganda, more than half of the scans were classified as inappropriate. ${ }^{5}$ Another study in Canada revealed that in the ten-year period between 1996 and 2006, the number of ultrasound scans per 1000 pregnancies increased by more than fifty per cent, with the increase being the same for both low-risk and high-risk pregnancies; in that study the proportion of pregnancies with at least four ultrasound examinations increased almost threefold, with the increase being more pronounced among low-risk pregnancies. ${ }^{6}$

The value of the use of ultrasound imaging for specific indications, such as detection of possible fetal malformation, placental localization and confirmation of multiple pregnancies, has been clearly shown. It helps with accurate assessment of gestational age, early detection of abnormal pregnancy, and fetal growth monitoring among several other advantages. $1,7,8,9$ 
It is also useful in the early detection and monitoring of the conditions that give rise to fetal and maternal compromise $^{9}$ and has become a useful adjunct to the management efforts of practitioners in this regard. In addition to the medical indications for antenatal ultrasonography some pregnant women have certain expectations of ultrasound examination; they may also want to or do actually have ultrasonography for various reasons. These include checking for fetal abnormalities, to see that all was normal, for their own reassurance, and assessing fetal growth. ${ }^{10,11}$ Others include checking for fetal viability, fetal sex determination, checking for number of fetuses, determination of gestational age and placental localization. ${ }^{2,7,11,12}$

Apart from the many medical benefits of antenatal ultrasound, the procedure is also known to have certain psychological effects. Generally, most mothers are happy when they see their fetuses on the monitor of the ultrasound machine, an indication of the viability of the pregnancy. There is a belief that it initiates bonding ${ }^{2}$. There is a fairly consistent reduction in levels of anxiety, depression, hostility and somatic symptoms after the ultrasound scan; it must be mentioned that the prospect of an ultrasound examination may increase anxiety levels beforehand and the reduction after the procedure reflects a return to baseline levels. ${ }^{13}$ The negative side of ultrasound use is when there are significant morphological defects or other unexpected findings like multiple pregnancies which may have adverse effects on the mother ${ }^{1,8,9}$ and may provoke an emotional crisis. ${ }^{14}$

Ultrasound is one of the many technologies that were initially developed in affluent parts of the world but are now proliferating in the developing world., ${ }^{1,8,9}$ There are so many ultrasound centres in Ghana (in both private and government facilities) and antenatal care practitioners are equally increasingly referring their clients for ultrasound evaluation of their pregnancies. A lot is known about women's views on and experiences of this technology in the developed world but not a lot of work has been done to find out how this new technology is perceived by women in a developing African country like Ghana.

The aim of the study was therefore to evaluate how Ghanaian women who have gone through pregnancy and have had live births, perceive the use of the technology, and their assessment of the experience, of antenatal ultrasound scanning.

\section{METHODS}

This study was a cross-sectional study conducted between $25^{\text {th }}$ February and $16^{\text {th }}$ April, 2011 in three public health facilities in Accra, Ghana namely Korle Bu Teaching hospital, (the largest tertiary hospital in
Ghana, in southern part of Accra), University Of Ghana Hospital, Legon (located east of Accra), and Ga South District Hospital, Weija (located west of Accra). Korle $\mathrm{Bu}$ Teaching has a yearly antenatal attendance of 49,000 with about 10,500 deliveries a year. Legon Hospital has an annual antenatal attendance of 1786 and conducts about 900 deliveries a year. Ga South District Hospital has a yearly antenatal attendance of 4800 and conducts about 1500 deliveries a year.

The total population size considered from the three health facilities was approximately 12,900 deliveries a year. Assuming an antenatal ultrasound rate of $70 \%,{ }^{1}$, a margin of error of $5 \%$ and a confidence interval of 95\% and using Epi info version 3.3.2 the approximate sample size for the study was 315 patients. We adjusted this figure for approximately 10\% nonparticipation and $5 \%$ non-response and the new sample size worked out to be 400 .

Simple random sampling technique was used to select respondents for the study after proportionally allocating the number of respondents that should be selected from each hospital based on annual deliveries of the various hospitals. We interviewed 30, 50 and 320 for Legon, Ga South and Korle Bu respectively. However we decided to stop after we obtained 337 patients who responded fully to the questions.

A total of 337 patients were interviewed; 25 from University Hospital, 40 from Ga South District Hospital and 272 from Korle Bu Teaching Hospital. These samples sizes were decided on based proportionately on the annual antenatal attendance in each institution.

The respondents were women who had just delivered at these health institutions and were waiting to be discharged. Only women who were able to carry their pregnancy to term and had healthy live babies were included. Respondents were selected using a simple random sampling method. Each morning patients who had carried their pregnancies to term and had delivered live babies in the preceding 24 hours were sequentially assigned numbers based on time of delivery.

A number of women from the different hospitals (7 from KBTH, 2 from Ga South, 1 from Legon) were randomly selected using computer-generated (Microsoft Excel 2007) random numbers each day. The nature of the study was adequately explained to those selected. Respondents who agreed to participate and signed an informed consent were interviewed using a semi-structured questionnaire with both open and close-ended questions. Once the required number of respondents for each institution was obtained the study ended for that site. 
Information gathered from the women included, age and parity, gestational age at first ultrasound scan, occupation, educational level, marital status and assessment of the cost of the scan amongst others.

Other relevant issues that were assessed included the following:

1. What motivates women to go for ultrasound scan during the antenatal period

2. What women's knowledge of uses/advantages and dangers associated with ultrasound use in pregnancy is and what information is provided by health care providers.

3. How women assess the ultrasound scanning experience in general.

4. How eager women are to know the sex of their fetuses and its effect on them.

The findings were then summarized into percentages and other ratios using Microsoft excel and SPSS 16 and the relevant conclusions drawn from them.

\section{RESULTS}

A total of 337 women were interviewed over the period out of which 25 were from University Hospital, 40 from Ga South District Hospital, and 272 from Korle $\mathrm{Bu}$ Teaching Hospital. None of the respondents selected refused to participate.

\section{Demographic characteristics}

The ages of the clients ranged between 16 years and 42 years; the mean age was 28.1 (SD 5.2) years. The parity of the clients ranged between 1 and 8 with the mean parity being 2.1(SD 1.1). Most of the respondents, $236(70 \%)$ were married, 55(16.3\%) were cohabiting, $45(13.4 \%)$ were single and one of the respondents $(0.3 \%)$ was divorced. One hundred and thirty seven $(40.7 \%)$ of the respondents had basic education, 96 (28.5\%) had secondary education, $41(12.2 \%)$ tertiary, $19(5.6 \%)$ vocational and one respondent $(0.3 \%)$ postgraduate education; 43 (12.8\%), had not had any formal education.

One hundred and thirty-seven $(40.7 \%)$ of the respondents were traders, followed by $87(25.8 \%)$ were artisans, $40(11.9 \%)$ were professionals and $17(5 \%)$ were students; $38(11.3 \%)$ were not in any form of employment. Eighteen respondents (5.3\%) had other forms of employment like being porters, factory hands and house helps.

\section{What motivated women to go for antenatal ultra- sound}

The women had had between 1 and 8 scans with a mean of 2.2 (SD 1.1). Three hundred and fourteen women $(93.2 \%)$ had 3 or fewer scans (Table 1$)$. The mean gestational age at which the respondents had their first scan was 4.5 (SD 1.9) months; most of them, $177(52.5 \%)$ had it before or around the fourth month of pregnancy.

Table 1 Number of Scans per Pregnancy

\begin{tabular}{|l|l|l|}
\hline $\begin{array}{l}\text { Number } \\
\text { of Scans }\end{array}$ & $\begin{array}{l}\text { No of Re- } \\
\text { spondents }\end{array}$ & Percent \\
\hline 1 & 90 & 26.7 \\
\hline 2 & 129 & 38.3 \\
\hline 3 & 89 & 26.4 \\
\hline 4 & 15 & 4.5 \\
\hline 5 & 8 & 2.4 \\
\hline 6 & 3 & 0.9 \\
\hline 7 & 2 & 0.6 \\
\hline 8 & 1 & 0.3 \\
\hline Total & $\mathbf{3 3 7}$ & $\mathbf{1 0 0 . 0}$ \\
\hline
\end{tabular}

All of the respondents went for scans because they had been referred by a doctor or midwife. For most of these respondents, $183(54.3 \%)$, the doctor or midwife indicated the reason for which they were asked to go for the scan. However $11(3.2 \%)$ had additional scans without referral. Ten of these went for scans to confirm their pregnancy before booking and one went for it to check the presentation of her fetus.

Table 2 Reasons for request of Scan investigation

\begin{tabular}{|l|l|l|}
\hline Reason For Referral & Respondents & $(\mathbf{\%})$ \\
\hline Check Presentation Of Fetus & 85 & 25.2 \\
\hline Assess Fetal Wellbeing & 79 & 23.4 \\
\hline Confirm Pregnancy & 20 & 5.9 \\
\hline Estimate Gestational Age & 18 & 5.4 \\
\hline Estimate Weight Of Baby & 4 & 1.2 \\
\hline Investigate Bleeding PV & 4 & 1.2 \\
\hline Assess Amniotic Fluid Volume & 3 & 0.9 \\
\hline Determine Number of Fetuses & 2 & 0.6 \\
\hline Exclude Fetal Abnormalities & 1 & 0.6 \\
\hline Investigate Pains in The Abdomen & 1 & 0.3 \\
\hline To rule out Uterine Fibroids & 1 & 0.3 \\
\hline Respondents did not know & 117 & 0.3 \\
\hline Total & $\mathbf{3 3 7}$ & 34.7 \\
\hline
\end{tabular}

Three hundred and twenty-one (95.3\%) of the respondents had the scan as requested because they felt it was a very important component of the antenatal care while the remaining $16(4.7 \%)$ had the scan as requested but they did not consider it an important component. 
Knowledge of the indications, advantages and dangers of ultrasound use in pregnancy

The two most common reasons for which respondents thought they were asked to go for scan were: to check the presentation of the fetus, 85 respondents $(25.2 \%)$ and to assess fetal well-being, 79 respondents (23.4\%). As many as 117 respondents (34.7\%) could not indicate the reason for which they were referred for the scan. (Table 2).

The three most common perceived advantages mentioned by the respondents were, determination of the sex of the fetus, 85 respondents $(25.2 \%)$, check the presentation of the fetus, 67 respondents $(19.9 \%)$ and assess fetal well-being, 65 respondents (19.3\%). Sixtyseven respondents (19.9\%) could not think of any advantages of ultrasound use in pregnancy. (Table 3) None of the patients knew of any dangers associated with the use of ultrasound in pregnancy.

Table 3 Perceived Benefit of Scan

\begin{tabular}{|l|l|l|}
\hline Advantages Of Scan & $\begin{array}{l}\text { Number of } \\
\text { Respondents }\end{array}$ & $\begin{array}{l}\text { Percent } \\
(\mathbf{\%})\end{array}$ \\
\hline Determine Sex & 85 & 25.2 \\
\hline Check Presentation Of Fetus & 67 & 19.9 \\
\hline Assess Fetal Wellbeing & 65 & 19.3 \\
\hline Confirm Pregnancy & 20 & 5.9 \\
\hline Estimate Gestational Age & 13 & 3.9 \\
\hline Exclude Fetal Abnormalities & 11 & 3.3 \\
\hline Determine Number of Fetuses & 4 & 1.2 \\
\hline Localisation of Placenta & 3 & .9 \\
\hline Confirm Fetal Viability & 2 & 0.6 \\
\hline Assess Amniotic Fluid Volume & 1 & .3 \\
\hline Estimate Weight of Fetus & 1 & .3 \\
\hline Do not know & 65 & 19.3 \\
\hline Total & $\mathbf{3 3 7}$ & $\mathbf{1 0 0 . 0}$ \\
\hline
\end{tabular}

\section{Women's assessment of the ultrasound experience} in general

The cost of the scan ranged between GHC10 and GHC 35 with the mean cost being GHC14.85 (SD5.24). Seventy-seven $(22.8 \%)$ women paid GHC 10 . One hundred and eighty respondents $(53.4 \%)$ thought the cost of the scan was high, while 157 (46.6\%) thought it was reasonable.

For $183(54.3 \%)$ women their healthcare givers (doctors and midwives) explained the reason for asking them to go for scan to them, while the remaining 154 $(45.7 \%)$ did not have any such explanation.
However after the scan 152(45.1\%) women had the results explained to them, while for $185(54.9 \%)$ no such explanations were given.

For the vast majority of the respondents, 239 women $(70.9 \%)$, the sonographer did not explain the procedure to them before the examination. Of the remaining 98 respondents $(29.1 \%)$ who had the procedure explained to them by the sonographer $92(93.9 \%)$ of these found the explanation helpful.

Only 89 respondents $(26.4 \%)$ were allowed to ask questions during the scanning process. For 160 respondents $(47.5 \%)$, the sonographer communicated the results of the scan to them at the end of the study; no such communication took place with the rest of the respondents.

Only 61 respondents $(18.1 \%)$ were allowed to see their fetus on the monitor. Forty-six of them $(75.9 \%)$ were excited about the experience, 4 (6.9\%) found the experience reassuring, one $(1.7 \%)$ felt more connected with their fetus, $4(6.9 \%)$ could not clearly see the features of the fetus on the monitor and $3(5 \%)$ did not understand what they saw. Two women $(3.4 \%)$ were indifferent about what they saw.

The general impression of the scanning experience by 308 respondents $(91.4 \%)$ was that ultrasound use in antenatal care was a good thing and should be encouraged. Twenty-nine respondents $(8.6 \%)$ did not see anything positive about the scanning experience.

Asked if they had any general comments about the experience other than what had been asked already, thirty-eight women $(10 \%)$ thought the scanning environment/atmosphere (both human and structures) was not friendly and $5(1.5 \%)$ thought the environment (sitting area, scanning room and bed sheets) was not neat enough.

The rest of the respondents did not have any major comments. Only $26(7.7 \%)$ respondents had their husbands with them during the scan. Asked whether they would want their husbands to be with them during ultrasound scan in their next pregnancy, most of the respondents $195(57.9 \%)$ responded in the affirmative.

Eagerness of women to know the sex of their fetus,

its effect on them. One hundred and sixty of the respondents (47.5\%) asked the sonographer for the sex of their fetus. For those who were told the sex of their fetus, $127(79.3 \%)$ were happy with the sex they were told their fetus had, $20(12.8 \%)$ were disappointed, 11 $(6.7 \%)$ were indifferent and $2(1.2 \%)$ doubted the results. 
For $138(86.5 \%)$, the sex of their baby at the time of delivery was the same as what the sonographer mentioned, while in the case of 22 respondents $(13.5 \%)$ it was not. Out of the respondents who found out the sex of their fetus was different from what the sonographer said, $10(47.1 \%)$ were happy because of successful delivery irrespective of sex, 7 (29.4\%) were surprised at the turn of events and $5(23.4 \%)$ were disappointed at the outcome.

\section{DISCUSSION}

Ultrasound technology is particularly important in antenatal surveillance in obstetrics where routine screening by ultrasound has become an integral part of antenatal care provision. The mean number of scans in this study was 2.2. This is identical to what was reported from Uganda ${ }^{5}$ and comparable to the 2.6 in Nottingham, $\mathrm{UK}^{15}$ and 2.55 in rural China ${ }^{16}$, but low compared to 5,5,6,6 and 5.9 reported from Syria, Hanoi (Vietnam) and Iran. ${ }^{7,9,17}$ It may also be mentioned that whereas in this study less than a tenth $(8.7 \%)$ of the respondents had 4 or more scans, in Hanoi two-thirds had more than four and a one-fifth had more than 10 scans. ${ }^{9}$

Some of the reasons that may have accounted for the situation in our environment were that most of the women thought that the cost of having a scan was high and also almost all the scans were done at the request of the doctor or midwife. This is in contrast to the situation in Hanoi where $30 \%$ of women went for scans without doctors' or midwives' referral, just to reassure themselves that their fetuses were fine.

Again, while in general doctors in Ghana would usually ask for a scan in uncomplicated pregnancies only when necessary, in other countries doctors in both private and public institutions asked pregnant women to attend each antenatal visit with a new scan report. ${ }^{9}$ It is also worth noting that all the women in this study attended antenatal clinic in public health institutions where there was no motivation for the doctors to overuse the ultrasound technology for their own financial gain as was the case in some facilities mentioned in similar studies in Syria and Hanoi. ${ }^{7,9}$

The mean gestational age for first scan for respondents in this study was 18 weeks (4.5 months). This is rather late compared to a mean of 11 weeks reported from Denmark $^{10}$ and 14.3 weeks from Nottingham (UK). ${ }^{15}$ The late gestational age in this study may be explained by the fact that generally in Ghana pregnant women book for antenatal care between four to five months of gestation and goes to support the fact that most women in the study will have scans only when they have been asked to so do by their health care providers.
The three most common perceived advantages of antenatal ultrasound mentioned by the respondents, determination of the sex of the fetus, checking the presentation of the fetus, and assessing fetal well-being, are comparable to findings made in studies in Ibadan (Nigeria), Uganda and Tanzania. ${ }^{2,12,18}$ It is significant to note, however, that whereas in studies in Karachi (Pakistan), Nnewi (Nigeria) and Japan detection of fetal abnormalities feature prominently among the top three uses of ultrasound mentioned by mothers ${ }^{11,19,20}$, in this study detection of fetal anomalies is much further down the order, with only about three percent being aware of it. This may be a reflection of poor knowledge of the occurrence of fetal abnormalities among the women.

The findings in this study suggest that there is inadequate flow of information from health care providers to the women concerning antenatal ultrasound. More than $45 \%$ of the women did not have any explanations given to them concerning the reasons for the ultrasound request. This is in contrast to the findings in Nottingham (UK) where for almost $85 \%$ of scans done respondents indicated that they had been provided at least one reason for the $\operatorname{scan}^{15}$. It is not surprising that in this study more than a third of respondents could not figure out why they had to have an ultrasound scan.

Even after the procedure almost $55 \%$ did not have the results explained to them by their doctors or midwives. This coupled with the fact that the sonographer did not communicate his/her findings to $52.5 \%$ of the women, suggests that more than half the women had no idea about the results of the procedure. It is possible that this lack of information may have accounted for the situation in which almost one in five of respondents did not appreciate any advantages of the use of ultrasound in pregnancy.

The experience of the ultrasound process itself could have been better: for most of the women $(70.9 \%)$, the sonographer did not explain the procedure to them before the examination, less than one-fifth were allowed to see their fetus on the monitor (which had positive effects on most of them) and just a little over a quarter were allowed to ask questions during the process. Visualization of the fetus on ultrasound has been a source of pleasure, comfort and emotional reassurance for pregnant women ${ }^{7,10,18,21}$ and has been reported to enhance feelings of bonding between women and their fetuses $22,23,24$. Ghanaian women should not be denied these psychological benefits.

The lack of opportunity to ask questions during the ultrasound procedure has also been reported from Uganda where women's questions were either not re- 
sponded to or were responded to rudely. This lack of communication led to most of the women being dissatisfied with the person doing the ultrasound. ${ }^{12}$ Communication between the sonographer and the client before, during and after the ultrasound examinations has been described as a major issue that influences women's ultrasound experience. This affects the client's cooperation during the procedure and her perception of the adequacy of the procedure at the end of the examination. ${ }^{1,7}$ Many centres take the initial counseling of the respondents before the procedure for granted especially because the examination is non-invasive. , $^{1,8,9}$ This education or information provision has another important role in that without adequate counseling and assurance, respondents with ambivalent or negative feelings about ultrasound scan tend not to enjoy the examination. ${ }^{7}$

It is important to mention, however, that in a systematic review comparing high feedback (in which women could see the screen and they received detailed explanations of the images) and low feedback (in which only the operator could see the screen and the women were told the results at the end) antenatal ultrasound, the findings did not show that women in the high feedback groups were more likely to choose very positive adjectives to describe their feelings after the scan. On the other hand they were more likely to act positively towards cessation of smoking and alcohol consumption during pregnancy. ${ }^{25}$

One major reason for the deficiency in communication between sonographer and clients in the present study may be the large numbers of patients sonographers have to deal with in a day. Lack of information has consistently been reported to be associated with increased fear and misconceptions of ultrasonography ${ }^{1,26}$ and therefore every effort must be made by health care workers to provide adequate information to women before, during and after antenatal ultrasound.

At the antenatal clinics, ultrasound and its place in the management of pregnancy should be added to the antenatal education topics; doctors and midwives should clearly explain to women the reasons for requesting ultrasound scans; sonographers must explain the procedures involved in performing ultrasound scan as well as allow the women to see their fetuses on the monitor and answer their questions in a sensitive manner.

Although only $7.7 \%$ respondents had their husbands with them during the scan, fifty-seven percent of all the women would want to have their husbands with them when they have scans in their next pregnancy. Some women see ultrasonography as a way of getting their husbands involved in childbearing, to enhance not only the father-child bond, but also the marital relationship.?
There is evidence that routine antenatal ultrasound scans has effects on the psychological well-being of both women and $\operatorname{men}^{27}$, and may also have significant effects on parental attitudes to each other and to the fetus $^{28}$. It is possible that the very low attendance by fathers in this study may be due to lack of knowledge that fathers could attend the ultrasound procedure. This call for efforts to be made by centres that provide ultrasound services to make special arrangements to enable husbands or partners be present during the procedure.

Knowing the sex of the fetus has been documented as one of the major motivations for which women go for antenatal scans. ${ }^{7,8,9,18}$ Reasons assigned for wanting to know fetal sex have included the desire for a particular gender, the sense of relief if the sex of the fetus matched their preferences, and to enable adequate preparation for the coming baby in terms of shopping. ${ }^{7,29,30 .}$ In societies with strong male preference fetal sex determination has been used for sex-selective abortions. $^{31}$ In this study $47.5 \%$ of the respondents wanted to know and were told the sex of their fetus. This is low when compared to $94.5 \%$ reported from Sokoto (Nigeria), and almost a hundred percent from Uganda $^{4,30}$; however, it is much higher than the $21.6 \%$ reported from Nnewi and 22.6\% from Ibadan (both in Nigeria). ${ }^{2,19}$

In the present study, $86.5 \%$ of fetal sex determination was correct at delivery. This is low compared to reported accuracy of $99-100 \%$ for second trimester fetuses without malformed external genitalia. ${ }^{32}$ It is significant to note that less than a quarter of those whose antenatal fetal sex assignment was different at delivery were actually disappointed. This suggests that even though Ghanaian women may want to know the sex of their fetuses, knowing that their babies are well, irrespective of the sex, is perhaps more important and reassuring to them.

The women in this study saw the ultrasound scan as an integral part of the antenatal care and thus considered it necessary for continuing attending antenatal clinic. This attitude may have been encouraged by the fact that none of them was aware of any dangers of ultrasound examinations, either to themselves or to their unborn babies.

It is important to note that about $10 \%$ of the clients thought the ultrasound scanning environment was not very friendly. This raises a cause for concern and it is therefore very important for managers of such facilities to ensure that they provide the right ambience and that members of staff exhibit the right attitudes towards clients who report for ultrasound scanning. 
In spite of some of the negative experiences in the process of having antenatal ultrasound scan, most (91.4\%) of the women saw the whole scan experience as a good one and were willing to go through it in their next pregnancy. It is possible that with a bit of effort to address the issues raised in this study almost all the women will want to have antenatal ultrasound scans in their next pregnancies.

\section{Limitations of the study}

It is possible that because only women with live babies were included in the study their negative experiences may have been under-reported due to the delight and excitement over the good outcomes to their babies.

\section{CONCLUSIONS}

1. Most Ghanaian women go for antenatal ultrasound scans because a doctor or midwife would have requested it.

2. Most women consider antenatal ultrasound an essential part of antenatal care.

3. The most common perceived advantages of ultrasound were determination of the sex of the fetus, checking the presentation of the fetus, and assessing fetal well-being.

4. The clients are not aware of any harmful effects of ultrasound.

5. There is inadequate information flow from doctors, midwives and sonographers to clients concerning the indications for the ultrasound, the processes involved and the results of the procedure.

6. Most women are not allowed to see their fetuses on the monitor.

7. Less than half of the women were told the sex of their fetuses.

\section{RECOMMENDATIONS}

a. Doctors and midwives should educate their patients on reasons why they ask them to go for ultrasound scans and should make every effort to explain the ultrasound findings to them.

b. Sonographers should take time to educate and communicate with their patients before, during and after the scan.

c. Health facilities that provide ultrasound services should make their environment more friendly to their patients both in terms of staff attitudes and in the physical surroundings.

d. Women should be encouraged to bring along their husbands/partners for the ultrasound scan if they so desire.

\section{REFERENCES}

1. Tautz S., Jahn A, Molokomme I., Görgen R.. Between fear and relief: how rural pregnant women experience fetal ultrasound in a Botswana district hospital. Social Science \& Medicine. 2000; 50: 689-701.

2. Enakpene C. A., Morhason-Bello I. O., Marinho A.O., Adedokun B.O., Kalejaiye A. O., Sogo K. et al. Clients' reasons for prenatal ultrasonography in Ibadan, South West of Nigeria. BMC Women's Health 2009; 9:12.

3. World Health Organisation, 1994. Mother Baby Package: Implementing Safe Motherhood in Countries, Maternal Health and Safe Motherhood Programme. WHO, Geneva.

4. Maaji S. M., Ekele B. A., Bello S. O., MorhasonBello I.O. Do women want disclosure of fetal gender during prenatal ultrasound scan? Ann Afr Med 2010; 9: 11-14.

5. Mubuuke A. G., Kiguli-Malwadde E., Businge F., Byanyima R. K. Utilisation of obstetric sonography at a peri-urban health centre in Uganda. Pan African Medical Journal 2011 7: 24

6. You J.J., Alter D. A., Stukel T.A., McDonald S.D., Laupacis A., Liu Y., Ray J. G. Proliferation of prenatal ultrasonography. CMAJ 2010; 182: 143151.

7. Bashoura H., Hafezb R., Abdulsalamc A. Syrian Women's Perceptions and Experiences of Ultrasound Screening in Pregnancy: Implications for Antenatal Policy. Reprod Health Matters. 2005; 13: $147-154$.

8. Garcia J., Bricker L., Henderson J., Martin A., Mugford M., Nielson J., Roberts T. Women's Views of Pregnancy Ultrasound: A Systematic Review. BIRTH 2002; 29: 225-250.

9. Gammeltoft T., Thi H., Nguyen T. The Commodification of Obstetric Ultrasound Scanning in Hanoi, Viet Nam. Reproductive Health Matters 2007; 15:163-171

10. Gudex C., Nielsen B. L., Madsen M. Why women want prenatal ultrasound in normal pregnancy. $\mathrm{Ul}$ trasound Obstet Gynecol. 2006; 27: 145-150.

11. Munim S., Khawaja N. A., Qureshi R. Knowledge and awareness of pregnant women about ultrasound scanning and prenatal diagnosis. J Pak Med Assoc. 2004; 54: 553-555.

12. Mubuuke A. G., Kiguli-Malwadde E., Byanyima R., Businge F. Current knowledge, attitudes and practices of expectant women toward routine sonography in pregnancy at Naguru health centre, Uganda. Pan Africa Medical Journal. 2009 3:18.

13. Ayers S., Pickering A.D. Psychological factors and ultrasound: differences between routine and highrisk scans. Ultrasound Obstet Gynecol. 1997; 9: 76-79.

14. Sommerseth E., Sundby J. Women's experiences when ultrasound examinations give unexpected 
findings in the second trimester. Women and Birth. 2010; 23: 111-116.

15. Whynes D. K. Receipt of information and women's attitudes towards ultrasound scanning during pregnancy. Ultrasound Obstet Gynecol. 2002;19: 7-12.

16. Huang K., Tao F., Raven J., Liu L., Wu X., Tang S. Utilization of antenatal ultrasound scan and implications for caesarean section: a cross sectional study in rural Eastern China. BMC Health Services Research. 2012 12:93.

17. Ranji A., Dykes A. K. Ultrasound screening during pregnancy in Iran: womens' expectations, experiences and number of scans. Midwifery. 2012; 28: 24-29.

18. Firth E. R., Mlay P., Walker R., Sill P. R. Pregnant women's beliefs, expectations and experiences of antenatal ultrasound in Northern Tanzania. African Journal of Reproductive Health. 2011; 15: 91108.

19. Igwegbe A.O., Ugboaja J.O., Monago E.N. Attitude and perceptions of women on routine antenatal ultrasound examination in Nnewi, South East, Nigeria. Journal of Biomedical Investigation. 2009; 7:

20. Murakami K., Tsujino K., Sase M., Nakata M., Ito M., Kutsunugi S. Japanese women's attitudes towards routine ultrasound screening during pregnancy. Nursing and Health Sciences 2012; 14: 95101.

21. Ohman S. G., Waldenström U. Second trimester routine ultrasound screening: expectations and experiences in a nationwide Swedish sample. Ultrasound Obstet Gynecol 2008; 32: 15-22.

22. Ekelin M., Crang-Svalenius E., Dykes A. K. A qualitative study of mothers' and fathers' experiences of routine ultrasound examination in Sweden. Midwifery 2004; 20: 335-344.

23. Boukydis C. F., Treadwell M. C., Delaney-Black V., Boyes K., King M., Robinson T., Sokol R. Women's responses to ultrasound examinations during routine screens in an obstetric clinic. $J$. $U l$ trasound Med. 2006; 25: 721-728.

24. Molander E., Alehagen S., Berterö C. M. Routine ultrasound examination during pregnancy: a world of possibilities. Midwifery 2010; 26: 18-26.

25. Nabhan A. F., Faris M.A. High feedback versus low feedback of prenatal ultrasound for reducing maternal anxiety and improving maternal health behaviour in pregnancy. Cochrane Database of Systematic Reviews 2010, Issue 4. Art. No.:CD007208. DOI:10.1002/14651858.CD007208.pub2.

26. Ekelin M., Crang-Svalenius E., Dykes A-K. Developing the P30R-U scale to measure parents' expectations, experiences and reactions to routine ultrasound examinations during pregnancy. $J$ Reprod Infant Psyc 2008; 26: 211-228.

27. Ekelin M., Crang Svalenius E., Larsson A. K., Nyberg P., Marsál K., Dykes A. K. Parental expectations, experiences and reactions, sense of coherence and grade of anxiety related to routine ultrasound examination with normal findings during pregnancy. Prenat Diagn. 2009; 29: 952-959.

28. Zlotogorski Z., Tadmor O., Rabinovitz R., Diamant Y. Parental attitudes toward obstetric ultrasound examination. $J$ Obstet Gynaecol Res. 1997; 23: 25-28.

29. Ekele B. A., Maaji S. M., Bello S. O., MorhasonBello I. O. Profile of women seeking fetal gender at ultrasound in a Nigerian obstetric population. Ultrasound 2008; 16: 199-202.

30. Mubuuke A.G. An exploratory study of the views of Ugandan women and health practitioners on the use of sonography to establish fetal sex. Pan African Medical Journal 2011 9: 36.

31. Garg S., Nath A. Female feticide in India: Issues and concerns. J. Postgrad Med. 2008; 54: 276-279.

32. Odeh M., Granin V., Kais M., Ophir E., Bornstein J. Sonographic fetal sex determination. Obstet $G y$ necol Surv. 2009; 64: 50-57. 